\title{
Preeclampsia: a link between trophoblast dysregulation and an antiangiogenic state
}

\author{
Roberto Romero, MD, DMedSci, ${ }^{1}$ and Tinnakorn Chaiworapongsa, MD1,2
}

\author{
1Perinatology Research Branch, Eunice Kennedy Shriver National Institute of Child Health and Human Development, \\ National Institutes of Health, Department of Health and Human Services, Bethesda, Maryland, USA, and Detroit, Michigan, USA. \\ 2Department of Obstetrics and Gynecology, Wayne State University School of Medicine, Detroit, Michigan, USA.
}

\begin{abstract}
A 24-year-old nulliparous woman developed mildly elevated blood pressure $(140-150 / 90-100 \mathrm{mmHg})$ without proteinuria $(20 \mathrm{mg}$ protein in a 24-hour urine collection) at $30^{6 / 7}$ weeks of gestation. The fetus was small for gestational age (estimated fetal weight under the fifth percentile). At $32^{5 / 7}$ weeks of gestation, the patient complained of epigastric pain, blood pressure was $180 / 110 \mathrm{mmHg}$, proteinuria was documented $(780 \mathrm{mg}$ protein in a 24-hour urine collection), schistocytes were detected in the peripheral smear, platelet count was 60,000 cells per $\mathrm{mm}^{3}$, and serum glutamic oxaloacetic transaminase was $234 \mathrm{U} / 1$. The patient was diagnosed with severe preeclampsia/HELLP syndrome. Antenatal steroids were administered to induce fetal lung maturity. She and her family want to know the causes of this condition, what treatment is available, and whether there are any longterm implications of this diagnosis.
\end{abstract}

\section{Current therapy}

The patient described above, with new-onset hypertension $(\geq 140 / 90 \mathrm{mmHg})$ and proteinuria ( $\geq 300 \mathrm{mg} / 24$-hour urine) after 20 weeks of gestation, meets the criteria for the diagnosis of preeclampsia (1-3). The presence of epigastric pain, thrombocytopenia, schistocytes, and elevated hepatic enzymes is characteristic of a severe form of preeclampsia, known as HELLP (hemolysis, elevated liver enzymes, and low platelet count) syndrome (4-9). Preeclampsia is a multisystemic disorder and a leading cause of maternal and perinatal morbidity/mortality (10). Maternal complications may include convulsions (eclampsia), intracranial hemorrhage, liver hematoma and rupture, pancreatitis, renal failure, disseminated intravascular coagulation, and maternal death. The only known treatment for preeclampsia is delivery of the fetus and placenta. Medical management consists of the administration of intravenous magnesium sulfate to decrease the risk of eclampsia (11) and antihypertensive agents (e.g., hydralazine and/or labetalol) to prevent the consequences of a hypertensive crisis (e.g., stroke). The decision to deliver a patient must balance the risks and benefits for the mother and fetus. In term ges-

Conflict of interest: The authors have declared that no conflict of interest exists.

Citation for this article: J Clin Invest. 2013; 123(7):2775-2777. doi:10.1172/JCI70431. tations, when the fetus is mature, the issue is straightforward; delivery will address the maternal syndrome without major risk to the neonate (12). In preterm gestations, however, the risk to the mother of continuing the pregnancy must be balanced with the risk of preterm birth ( $<37$ weeks of gestation), which is associated with an increased risk of short-term complications largely attributed to multiple organ immaturity (e.g., respiratory distress syndrome, intraventricular hemorrhage, necrotizing enterocolitis, neonatal sepsis) and longterm complications (e.g., chronic lung disease, neurodevelopmental disorders).

Preeclampsia is considered an inexorable disorder, and the only definitive treatment is delivery. Current medical therapy after the diagnosis of preeclampsia is aimed at preventing maternal and fetal complications (e.g., magnesium sulfate, antihypertensive agents, and antenatal steroids). Yet, there are reasons to be optimistic. Prevention of this disorder is possible. Aspirin administration in patients with a prior history of preeclampsia reduces the rate of recurrence by $10 \%$ (13). Moreover, a recent trial indicates that the combination of nitric oxide donors and antioxidants (L-arginine and vitamins $\mathrm{E}$ and $\mathrm{C}$ ) may reduce the recurrence rate by $17 \%$ (14). Given the modest effect size of these interventions, a continued search for effective interventions is needed. The identification of preventive and therapeutic strategies can be guided by an improved understanding of the molecular mechanisms of disease operating in this syndrome. Development of a molecular taxonomy of disease would be a major step forward. It is now clear that preeclampsia is not a single entity but rather a syndrome with multiple etiologies. Therefore, biomarkers, preventive strategies, and treatment must be targeted to the specific mechanism of disease in each of the clusters of the syndrome.

Central to the pathophysiology of preeclampsia is ischemia of the placenta $(15,16)$, which leads to the release of soluble factors and micro/nanovesicles $(17,18)$ into the maternal circulation responsible for the clinical signs of preeclampsia: hypertension and proteinuria. These factors have been referred to as "toxins," hence the name "toxemia of pregnancy" (19). The cause of placental ischemia is thought to be inadequate remodeling of the spiral arteries that brings maternal blood into the intervillous space of the placenta $(20,21)$. The mechanism responsible for the remodeling involves migration of trophoblasts from the anchoring villi into the decidua and superficial myometrium as well as the lumen and wall of the spiral arteries (21-24).

Ten years ago, Maynard et al. reported that an antiangiogenic factor, soluble VEGFR-1 (sVEGFR-1) or soluble fms-like tyrosine kinase 1 (sFlt-1), was increased in the placentas of women with preeclampsia and that increased concentrations could recapitulate the features of the syndrome in pregnant rats (25). sVEGFR-1 plasma/ serum concentrations correlated with the severity of preeclampsia (26), and the elevation could be demonstrated prior to the clinical diagnosis (27). The observation that the sera of women with preeclampsia (but not that of normal pregnant women) inhibited angiogenesis in vitro suggested that the condition is associated with an antiangiogenic state. Importantly, administration of recombinant VEGF-121 attenuated hypertension and improved renal lesions in an animal model of preeclamp- 
sia generated by the overexpression of sVEGFR-1 (28). The antiangiogenic state of preeclampsia has been attributed to a relative deficit of angiogenic factors, such as VEGF and placental growth factor (PlGF), and an excess of antiangiogenic factors, such as sVEGFR-1 and soluble endoglin (sEng). HELLP syndrome can be generated in pregnant rats through a combined excess of sVEGFR-1 and sEng (29). These soluble factors are excellent candidates to be some of the "toxins" produced by the placenta and responsible, at least in part, for some of the manifestations of preeclampsia (30-33).

\section{Research advances}

A fundamental question is whether there is a link between placental ischemia and the excess production of antiangiogenic factors in preeclampsia. During normal pregnancy, uterine blood flow increases to perfuse the intervillous space of the placenta and support fetal growth. This is made possible through a unique phenomenon, physiologic transformation of the spiral arteries, whereby trophoblasts (fetal cells) invade the decidua and superficial myometrium (maternal tissues) as well as the walls and lumen of the spiral arteries (34). The consequence is that the spiral arteries increase in diameter and the muscle of the media is destroyed, rendering the vessels unresponsive to vasoactive agents. In preeclampsia, trophoblast invasion is shallow and there is failure of physiologic transformation of the spiral arteries, which is thought to account for the decreased uteroplacental blood flow, although other mechanisms have been implicated (35). A role for trophoblasts, decidua, and immune mechanisms has been proposed to account for the disorder of deep placentation in preeclampsia (36).

Susan Fisher's laboratory has made major contributions to the understanding of the mechanisms responsible for normal and abnormal invasion of trophoblasts as well as the discovery that cytotrophoblasts adopt an endothelial adhesive phenotype within the lumen of the spiral arteries; this process is defective in preeclampsia $(22-24,30)$. A paper published by the Fisher group in this issue of the JCI identifies a protein that links two major pathologic processes implicated in the pathophysiology of preeclampsia: defective trophoblast invasion and an antiangiogenic state (37). Zhou et al. compared the cytotrophoblast transcriptomes isolated from patients with preeclampsia to those of a control group with spontaneous preterm labor/delivery. Many of the initial differences observed in the cultured cells were reverted to control levels after 48 hours in culture. The authors propose that this is evidence that the cytotrophoblast phenotypic alterations in preeclampsia are reversible and recovery possible. Demonstration that this is the case in vivo would be an exciting prospect, as it implies that treatment would be feasible. There are also immediate implications. Many scientists believe that when trophoblasts obtained from patients with a disease state (such as preeclampsia) are placed in culture, the cells retain the functional characteristics they had in vivo. The results of Zhou et al. suggest that this is not always the case, and future work needs to consider whether lack of differences in gene expression and other properties may represent an in vitro phenomenon.

Zhou et al. report for the first time upregulation of SEMA3B by trophoblast cells of patients with preeclampsia (37). This gene encodes semaphorin $3 \mathrm{~B}$, a protein known to induce apoptosis of tumor cells, and interferes with angiogenesis by binding to several members of the VEGF family (38). The authors reasoned that this molecule could play a key role in preeclampsia by inhibiting trophoblast invasion through apoptosis and contributing to the antiangiogenic state observed in the syndrome. The evidence in support of this includes: (a) SEMA3B mRNA and protein are overexpressed in trophoblasts from patients with preeclampsia; (b) the receptors NRP-1 and NRP-2 are expressed in villous and invasive cytotrophoblasts, creating conditions for an autocrine loop; and (c) SEMA3B reduces cytotrophoblast invasion by $60 \%$ through the induction of apoptosis and inhibits angiogenesis in a chick chorioallantoic membrane bioassay. These effects are mediated by opposing VEGF signaling through inhibition of PI3K (preventing the association of p85 and $\mathrm{p} 110 \alpha)$. Zhou et al. describe the operative intracellular signaling mechanisms and propose a pathway for the pathogenesis of preeclampsia (see Figure 6 of Zhou et al.; ref. 37).

\section{Recommendations}

The case for SEMA3B can be strengthened by demonstrating that the administration of this protein (directly or through a vector) to pregnant animals can induce hypertension, proteinuria, and other features of this syndrome (e.g., fetal growth restric- tion, thrombocytopenia, hepatic dysfunction, renal lesions). This should be reversible by blocking the effects of SEMA3B in vivo. Proof of a link between a trophoblast disorder and preeclampsia also requires evidence that overexpression of SEMA3B results in shallow placentation and failure of physiologic transformation of the spiral arteries. It would be important to determine whether the circulating concentrations of SEMA3B differ between patients with preeclampsia and normal pregnant women and whether such differences correlate with the severity of the disease and are detectable prior to clinical diagnosis. Demonstrating to what degree the putative involvement of SEMA3B is specific to preeclampsia is also desirable. An abnormal antiangiogenic profile, characterized by low concentrations of PlGF and high concentrations of the antiangiogenic molecules sVEGFR-1 and sEng, was discovered in the context of preeclampsia, but we now know that such abnormalities can also be observed in patients destined to have fetal growth restriction (39) as well as fetal death (both without preeclampsia) $(40,41)$ and a subset of patients with spontaneous preterm labor (42). These syndromes have also been shown to feature failure of physiologic transformation of the spiral arteries, a disorder of deep placentation (43). However, even if specificity could not be proven because derangements in SEMA3B expression were to occur in other obstetrical syndromes, this would be welcome news because it would expand the importance of this novel pathway in pregnancy complications. In our view, the crucial issue is the mechanism of disease, rather than the current diagnostic taxonomy of obstetrical diseases. In other words, if a specific pathway (e.g., SEMA3B or others) leads to an antiangiogenic state and disorders of deep placentation and such derangement could be identified in early pregnancy (or even before), there would be opportunities for the prevention of preeclampsia and other adverse pregnancy outcomes, which have proven to be virtually intractable for more than a century.

Finally, the patient and the family referenced in the clinical vignette would like to know the implications of this diagnosis for future health and disease. Patients with preeclampsia are at a greater risk for essential hypertension (44), cardiovascular diseases (including myocardial infarction, stroke, etc.) (45), type 2 diabetes (46), and chronic renal disease later in life (47). Thus, 
pregnancy can be viewed as a multisystem "stress" test, and the occurrence of preeclampsia may represent indirect evidence that vascular abnormalities may be at play. This concept has major healthcare implications, because prevention of cardiovascular disease in women can begin after the diagnosis of preeclampsia.

\section{Acknowledgments}

This work was supported, in part, by the Perinatology Research Branch, Division of Intramural Research, Eunice Kennedy Shriver National Institute of Child Health and Human Development, National Institutes of Health, Department of Health and Human Services (NICHD/NIH) and, in part, with federal funds from NICHD, NIH, under contract no. HSN275201300006C.

Address correspondence to: Roberto Romero, Perinatology Research Branch, NICHD, NIH, DHHS, 3990 John R, 4 Brush South, Detroit, Michigan 48201, USA. Phone: 313.993.2700; Fax: 313.993.2694; E-mail: romeror@mail.nih.gov.

1. Lindheimer MD, Roberts JM, Cunningham FG, Chesley L. Introduction, history, controversies and definitions. In: Lindheimer MD, Roberts JM, Cunningham FG, eds. Chesley's Hypertensive Disorders in Pregnancy. San Diego, California, USA: Elsevier; 2009:1-24.

2. Steegers EA, von Dadelszen P, Duvekot JJ, Pijnenborg R. Pre-eclampsia. Lancet. 2010;376(9741):631-644.

3. Sibai B, Dekker G, Kupferminc M. Pre-eclampsia. Lancet. 2005;365(9461):785-799.

4. Weinstein L. Syndrome of hemolysis, elevated liver enzymes, and low platelet count: a severe consequence of hypertension in pregnancy. Am J Obstet Gynecol. 1982;142(2):159-167.

5. Romero R, et al. Clinical significance, prevalence, and natural history of thrombocytopenia in pregnancy-induced hypertension. Am J Perinatol. 1989;6(1):32-38.

6. Martin JN Jr, Blake PG, Perry KG Jr, McCaul JF, Hess LW, Martin RW. The natural history of HELLP syndrome: patterns of disease progression and regression. Am J Obstet Gynecol. 1991; 164(6 pt 1):1500-1509.

7. Sibai BM. Diagnosis, controversies, and management of the syndrome of hemolysis, elevated liver enzymes, and low platelet count. Obstet Gynecol. 2004;103(5 pt 1):981-991.

8. Romero R, et al. Clinical significance of liver dysfunction in pregnancy-induced hypertension. Am J Perinatol. 1988;5(2):146-151.

9. Roberts JM, Redman CW. Pre-eclampsia: more than pregnancy-induced hypertension. Lancet. 1993; 341(8858):1447-1451.

10. Khan KS, Wojdyla D, Say L, Gulmezoglu AM, Van Look PF. WHO analysis of causes of maternal death: a systematic review. Lancet. 2006; 367(9516):1066-1074.

11. [No authors listed]. Which anticonvulsant for women with eclampsia? Evidence from the Collaborative Eclampsia Trial. Lancet. 1995;345(8963):1455-1463.

12. Koopmans CM, et al. Induction of labour versus expectant monitoring for gestational hypertension or mild pre-eclampsia after 36 weeks' gestation (HYPITAT): a multicentre, open-label randomised controlled trial. Lancet. 2009;374(9694):979-988.

13. Askie LM, Duley L, Henderson-Smart DJ, Stewart LA. Antiplatelet agents for prevention of pre-eclampsia: a meta-analysis of individual patient data. Lancet. 2007;369(9575):1791-1798.

14. Vadillo-Ortega F, et al. Effect of supplementation during pregnancy with L-arginine and antioxidant vitamins in medical food on pre-eclampsia in high risk population: randomised controlled trial. $B M J$. 2011;342:d2901.

15. Young J. The aetiology of eclampsia and albuminuria and their relation to accidental haemorrhage: (an anatomical and experimental investigation.). Proc $R$ Soc Med. 1914;7(Obstet Gynaecol Sect):307-348.

16. Ogden E, Hildebrand GJ, Page EW. Rise of blood pressure during ischemia of the gravid uterus. Proc Soc Exp Biol Med. 1940;43(1):49-51.

17. Redman CW, et al. Review: Does size matter? Placental debris and the pathophysiology of pre-eclampsia. Placenta. 2012;33(suppl):S48-S54.

18. Tannetta DS, Dragovic RA, Gardiner C, Redman $\mathrm{CW}$, Sargent IL. Characterisation of syncytiotrophoblast vesicles in normal pregnancy and pre-eclampsia: expression of Flt-1 and endoglin. PloS One. 2013;8(2):e56754.

19. Romero R, Lockwood C, Oyarzun E, Hobbins JC. Toxemia: new concepts in an old disease. Semin Perinatol. 1988;12(4):302-323.

20. Brosens IA, Robertson WB, Dixon HG. The role of the spiral arteries in the pathogenesis of preeclampsia. Obstet Gynecol Annu. 1972;1:177-191.

21. Pijnenborg R, Brosens I. Deep trophoblast invasion and spiral artery remodeling. In: Pijnenborg R, Brosens I, Romero R, eds. Placental Bed Disorders: Basic Science and its Translation to Obstetrics. New York, New York, USA: Cambridge Press; 2011:97-108.

22. Zhou Y, Damsky CH, Chiu K, Roberts JM, Fisher SJ. Preeclampsia is associated with abnormal expression of adhesion molecules by invasive cytotrophoblasts. J Clin Invest. 1993;91(3):950-960.

23. Zhou Y, Damsky CH, Fisher SJ. Preeclampsia is associated with failure of human cytotrophoblasts to mimic a vascular adhesion phenotype. One cause of defective endovascular invasion in this syndrome? J Clin Invest. 1997;99(9):2152-2164.

24. Zhou Y, et al. Human cytotrophoblasts adopt a vascular phenotype as they differentiate. A strategy for successful endovascular invasion? J Clin Invest. 1997; 99(9):2139-2151.

25. Maynard SE, et al. Excess placental soluble fmslike tyrosine kinase 1 (sFlt1) may contribute to endothelial dysfunction, hypertension, and proteinuria in preeclampsia. J Clin Invest. 2003; 111(5):649-658.

26. Chaiworapongsa T, et al. Evidence supporting a role for blockade of the vascular endothelial growth factor system in the pathophysiology of preeclampsia. Young Investigator Award. Am J Obstet Gynecol. 2004;190(6):1541-1547.

27. Levine RJ, et al. Circulating angiogenic factors and the risk of preeclampsia. $N$ Engl J Med. 2004; 350(7):672-683.

28. Li Z, et al. Recombinant vascular endothelial growth factor 121 attenuates hypertension and improves kidney damage in a rat model of preeclampsia. Hypertension. 2007;50(4):686-692.

29. Venkatesha $S$, et al. Soluble endoglin contributes to the pathogenesis of preeclampsia. Nat Med. 2006; 12(6):642-649.

30. Zhou Y, et al. Vascular endothelial growth factor ligands and receptors that regulate human cytotrophoblast survival are dysregulated in severe preeclampsia and hemolysis, elevated liver enzymes, and low platelets syndrome. Am J Pathol. 2002; 160(4):1405-1423.
31. Luttun A, Carmeliet P. Soluble VEGF receptor Flt1: the elusive preeclampsia factor discovered? J Clin Invest. 2003;111(5):600-602.

32. Gilbert JS, Babcock SA, Granger JP. Hypertension produced by reduced uterine perfusion in pregnant rats is associated with increased soluble fms-like tyrosine kinase-1 expression. Hypertension. 2007; 50(6):1142-1147

33. Ahmed A. New insights into the etiology of preeclampsia: identification of key elusive factors for the vascular complications. Thromb Res. 2011; 127(suppl 3):S72-S75

34. Brosens I, Robertson WB, Dixon HG. The physiological response of the vessels of the placental bed to normal pregnancy. J Pathol Bacteriol. 1967; 93(2):569-579.

35. Burton GJ, Woods AW, Jauniaux E, Kingdom JC. Rheological and physiological consequences of conversion of the maternal spiral arteries for uteroplacental blood flow during human pregnancy. Placenta. 2009;30(6):473-482.

36. Hiby SE, et al. Combinations of maternal KIR and fetal HLA-C genes influence the risk of preeclampsia and reproductive success. J Exp Med. 2004; 200(8):957-965.

37. Zhou Y, et al. Reversal of gene dysregulation in cultured cytotrophoblasts reveals possible causes of preeclampsia. J Clin Invest. 2013;123(7):2862-2872.

38. Castro-Rivera E, Ran S, Thorpe P, Minna JD. Semaphorin 3B (SEMA3B) induces apoptosis in lung and breast cancer, whereas VEGF165 antagonizes this effect. Proc Natl Acad Sci U S A. 2004; 101(31):11432-11437.

39. Chaiworapongsa T, et al. The maternal plasma soluble vascular endothelial growth factor receptor-1 concentration is elevated in SGA and the magnitude of the increase relates to Doppler abnormalities in the maternal and fetal circulation. J Matern Fetal Neonatal Med. 2008;21(1):25-40.

40. Romero R, et al. An imbalance between angiogenic and anti-angiogenic factors precedes fetal death in a subset of patients: results of a longitudinal study. J Matern Fetal Neonatal Med. 2010;23(12):1384-1399.

41. Chaiworapongsa $\mathrm{T}$, et al. Maternal plasma concentrations of angiogenic/antiangiogenic factors in the third trimester of pregnancy to identify the patient at risk for stillbirth at or near term and severe late preeclampsia. Am J Obstet Gynecol. 2013; 208(4):287.e1-287.e15.

42. Chaiworapongsa $\mathrm{T}$, et al. A subset of patients destined to develop spontaneous preterm labor has an abnormal angiogenic/anti-angiogenic profile in maternal plasma: evidence in support of pathophysiologic heterogeneity of preterm labor derived from a longitudinal study. J Matern Fetal Neonatal Med. 2009;22(12):1122-1139.

43. Brosens I, Pijnenborg R, Vercruysse L, Romero R. The "Great Obstetrical Syndromes" are associated with disorders of deep placentation. Am J Obstet Gynecol. 2011;204(3):193-201.

44. Bellamy L, Casas JP, Hingorani AD, Williams DJ. Pre-eclampsia and risk of cardiovascular disease and cancer in later life: systematic review and meta-analysis. BMJ. 2007;335(7627):974.

45. McDonald SD, Malinowski A, Zhou Q, Yusuf S, Devereaux PJ. Cardiovascular sequelae of preeclampsia/eclampsia: a systematic review and meta-analyses. Am Heart J. 2008;156(5):918-930.

46. Callaway LK, Lawlor DA, O'Callaghan M, Williams GM, Najman JM, McIntyre HD. Diabetes mellitus in the 21 years after a pregnancy that was complicated by hypertension: findings from a prospective cohort study. Am J Obstet Gynecol. 2007; 197(5):492.e1-492.e7.

47. Vikse BE, Irgens LM, Leivestad T, Skjaerven R, Iversen BM. Preeclampsia and the risk of end-stage renal disease. NEngl J Med. 2008;359(8):800-809. 